\title{
Smart Phone-Based Automatic QT Interval Measurement
}

\author{
ET Lim, X Chen, CT Ho, ZK Tin, M Sankaranarayanan \\ Institute for Infocomm Research, Singapore
}

\begin{abstract}
A smart phone-based automatic QT interval measurement system was developed. The system can assist pharmaceutical company in QT prolongation assessment prior to new drug approval. The ECG signal is captured by wearable sensor and processed on the smart phone. The processed results are sent using cellular network to the internet server. Cardiologist can quickly analyze the results. It shortens the time for data collection. Besides, it is convenient for the test subjects as they do not need to visit the lab frequently.

The embedded algorithm on smart phone first detects QRS complex using slope-based method. Next the $Q$-wave onset and T-wave offset are detected using wavelet-based method. Subsequently, heart rate and QT interval are calculated. We combined the robustness of slope-based QRS detector and accuracy of wavelet-based feature point detector.

The algorithm and user interface have been implemented on a smart phone with ARM OMAP 730 processor running at 200MHz. Alive Technologies ECG sensor with Bluetooth communication is used to capture the ECG signal. The QT interval measurement system runs in real-time.
\end{abstract}

\section{Introduction}

QT interval is an important ECG diagnostic parameter for cardiologist [1]. Prolonged QT interval on the ECG is associated with an increased risk for arrhythmia and sudden death. On the other hand, it is found that many drugs can prolong the QT interval, especially if used in combination with other substances which affect their metabolism. Women are more prone to prolongation of the QT interval. If a drug prolongs the QT interval beyond normal limits, it could increase the risk of potentially life-threatening arrhythmias [2]. Therefore, some drug regulatory bodies are concerns with regard to drug-induced cardiac repolarization and QT interval data have become part of standard safety packages across the pharmaceutical industry.

Here we present a smart phone-based automatic QT interval measurement system. The system can assist pharmaceutical company in QT prolongation assessment prior to new drug approval. The smart phone receives ECG data from a wearable ECG sensor via Bluetooth connection and calculate the QT interval in real-time. The results are sent using cellular network to the internet server. Cardiologist can quickly access the data through internet browser. It shortens the time for data collection. Besides, it is convenient to the test subjects as they do not need to visit the lab frequently.

\section{Methods}

The embedded algorithm on smart phone first detects QRS complex using slope method [3]. Based on the detected R-peak and average RR-interval, the search boundaries for Q-wave onset and T-wave offset are defined separately. Within these boundaries, Q-wave onset and T-wave offset are detected using wavelet-based method [4]. Local averaging value is used to reduces random noise resulted from poor electrode contact while searching for the T-wave offset. Heart rate and QT interval are derived from the detected characteristic points. Figure 1 depicts the overall algorithm flow. Qon and $\mathrm{T}_{\text {off }}$ denote $\mathrm{Q}$-wave onset and $\mathrm{T}$-wave offset respectively.

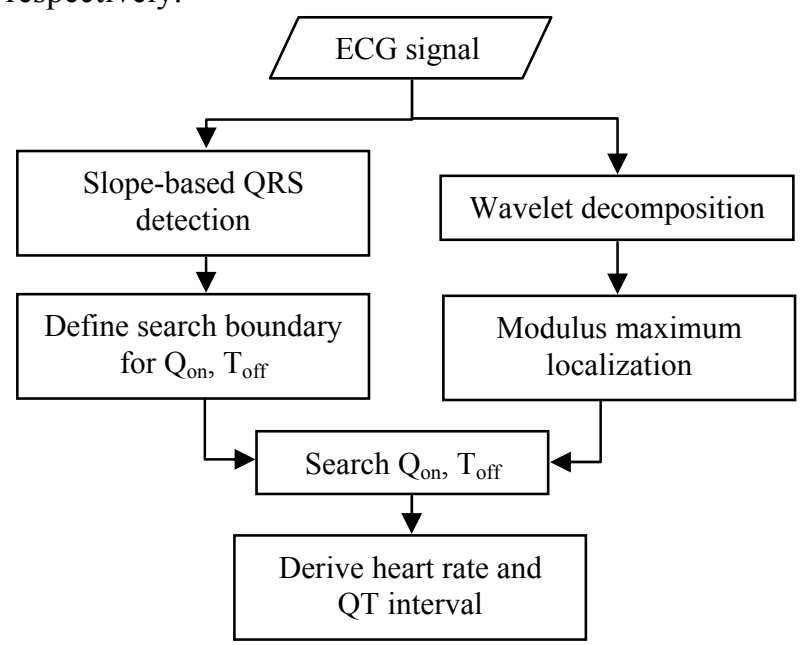

Figure 1. Flow chart for QT measurement and heart rate

Slope-based QRS complex detection algorithm is used here because it is more robust compared to wavelet-based method under real-time processing scenario. We compared these two QRS complex detection algorithms 
on offline and online ECG signal. During offline test, wavelet-based method achieves higher sensitivity $(99.75 \%)$ and positive predictivity $(99.85 \%)$ compare to slope-based method which achieve $99.38 \%$ sensitivity and $99.83 \%$ positive predictivity. However, during online test, slope-based method is able to detect QRS complex for low and changing R-peak amplitude. The waveletbased method has high false detection rate when R-peak magnitude is low and fluctuating. Despite having lower sensitivity and positive predictivity, slope-based QRS complex detection method is more suitable for online ECG signal. Besides, the computational complexity of slope-based QRS complex detection method is low.

\subsection{Wavelet Transform}

The wavelet transform of a function, $f$, with respect to a given mother wavelet, $\Psi$, is defined as,

$$
w_{s} f(x)=f * \psi_{s}(x)=\frac{1}{s} \int_{\infty}^{\infty} f(t) \psi\left(\frac{x-t}{s}\right) d t
$$

where $w_{s}$ is the wavelet transform operator and $s$ is the scale factor. Let $s=2^{i}$ ( $i \in Z$, and $Z$ denotes the integral set), then the wavelet transform is called dyadic wavelet transform. The dyadic wavelet transform can be calculated as:

$$
\begin{aligned}
& S_{2^{i}} f(n)=\sum_{k \in Z} h_{k} s_{2^{i-1}} f\left(n-2^{i-1} k\right) \\
& W_{2^{i}} f(n)=\sum_{k \in Z} g_{k} s_{2^{i-1}} f\left(n-2^{i-1} k\right)
\end{aligned}
$$

where $S_{2}{ }^{i}$ is a smoothing operator, and $W_{2}^{i}$ is the wavelet transform of digital signal $f(n)$. On the other hand, $h_{k}$ and $g_{k}$ are the coefficients of the corresponding low pass and high pass filters.

The close relationship between perfectly reconstructing filter banks and dyadic wavelets leads to computational efficiency. In this method the wavelet transform is implemented with a series of cascaded lowpass and high-pass filters following the approach of Mallat algorithm [5]. And to keep the time-invariance and the temporal resolution at different scales, we applied the algorithme a atrous [6] to keep same sampling rate in all scales.

\subsubsection{Q-wave onset detection}

Normally the onset of the QRS complex contains the high-frequency components, which are detected at finer scales, here we use scale $S^{2}$. By analyzing the decompose signal at scale $S^{2} \mathrm{Q}$-wave onset can be detected. Given Rpeak location detected by slope-based QRS complex detector, search backward within predefined boundary to locate the minimal point. From this minimal point, search backward further within predefined boundary to locate the maximal point. This maximal point in decompose signal at scale $S^{2}$ corresponds to Q-wave onset in the raw signal.

\subsubsection{T-wave offset detection}

The process for T-wave offset detection and delineation is given as follows: first of all, we define a search boundary for each beat, relative to the R-peak position and depending on a recursively computed RRinterval. Within this boundary, we look for local maxima. If two of them exceed a threshold, a T-wave is considered to be present. In this case, the local maxima of wavelet transform with amplitude greater than some threshold $\lambda T$ are considered as significant slopes of the wave, and the zero crossings between them as the wave peaks. And the offset of $\mathrm{T}$-wave is the ending of the maximum module pair.

\subsubsection{System implementation}

The algorithm has been implemented on $\mathrm{O}_{2}$ XPhone IIm running Windows Mobile 2003 Smart Phone Edition. This smart phone uses ARM OMAP 730 processor running at $200 \mathrm{MHz}$. Besides it has a Bluetooth connectivity to connect wirelessly with Alive Technologies ECG sensor which is used to capture the ECG data. Figure 2 depicts the $\mathrm{O}_{2}$ smart phone and the Alive Technologies ECG sensor.

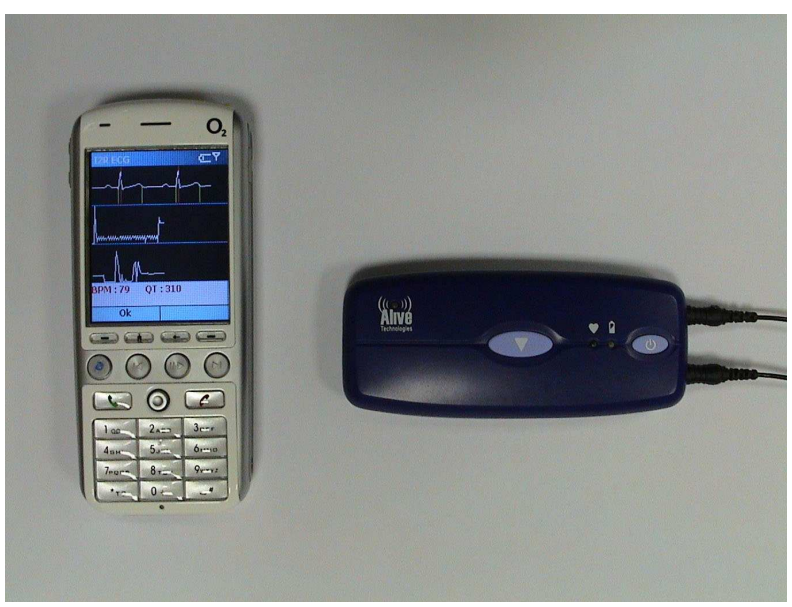

Figure 2. $\mathrm{O}_{2}$ smart phone and Alive Technologies ECG sensor

Microsoft Visual Studio 2005 tool is used to develop the algorithm. In order to speed up development time, we have used Microsoft Foundation Class. Graphic User Interface design and programming can be done quickly. However, we have less control over the system. The display cannot update fast enough with the data rate at 
$300 \mathrm{~Hz}$. Therefore, we reduced the display update rate to $100 \mathrm{~Hz}$. The update rate is sufficiently fast and ECG signal appears smooth. The data processing rate still maintains at $300 \mathrm{~Hz}$. The algorithm detects Q-wave onset, R-peak and T-wave offset in real-time. The ECG signal with annotated R-peak, Q-wave onset and T-wave offset is display on the smart phone as shown in Figure 3.

In this implementation, each computational cycle required approximately 12 multiplications, 6 divisions, 101 additions and 34 subtraction operations. With $300 \mathrm{~Hz}$ data rate, the smart phone is capable of processing the data in real-time.

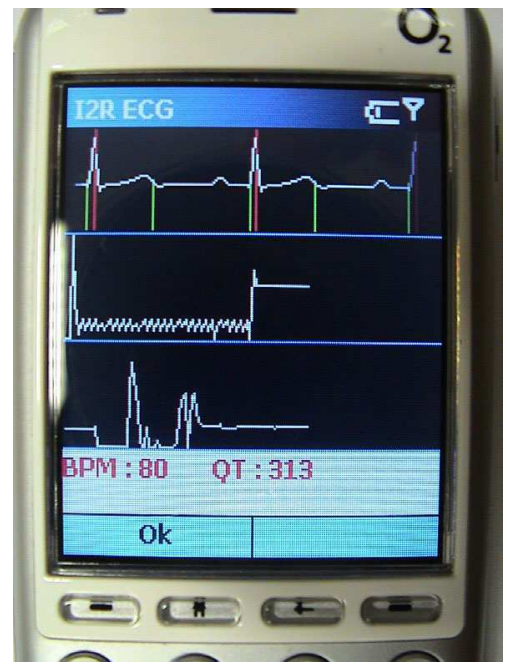

Figure 3. Data display on smart phone. Top row: ECG signal with annotated QRS peak (red line), Q-wave onset (green line) and $\mathrm{T}$-wave offset (green line). Middle row: Heart rate variation. Bottom row: Movement intensity as derive from accelerometer embedded in ECG sensor. BPM and QT denote heart rate (in beats per minute) and QT interval (in milliseconds) respectively.

\section{Results}

We have compared our Q-wave onset and T-wave offset detection algorithm with manual detection by cardiologist on QT database [7]. The database consist 105 fifteen minutes records. The database is composed from existing database including MIT-BIH Arrhythmia Database, European Society of Cardiology ST-T Database and Boston's Beth Israel Deaconess Medical Center ECG database. The database includes ECG records from subjects with normal sinus rhythm, arrhythmia, sudden death and other cardiac problems. For each record, around 30 beats has been manually annotated by at least one expert. We are interest in comparing the detection of Q-wave onset and T-wave offset. We compare individual beat by beat and calculate the mean and standard deviation of the differences.
Several QT measurement algorithms ware proposed in Computers in Cardiology Conference 2006 Challenge [8], [9]. In this challenge, participants are provided with 549 ECG records and algorithms were developed to identify the representative beat and provide a QT measurement result for each record. Each record consists of 15 simultaneous signals. The best score achieved by an automated method was $16.34 \mathrm{~ms}$, submitted by Dieter Hayn of ARC Seibersdorf Research GmbH, Graz, Austria [10]. In this method the characteristic points in all 15 signals are detected separately. After that the detection results are weighted and combined to produce the final measurement. Accuracy and reliability improved as more signals were used. However, in our application the Alive ECG sensor produces only single channel signal. Therefore, in our simulation study we use one signal to mimic the real application scenario. The accuracy and reliability of our algorithm will be lower compare to algorithm which uses multiple signals.

\begin{tabular}{|l|r|r|r|}
\hline & Beats & mean & $\begin{array}{r}\text { standard } \\
\text { deviation }\end{array}$ \\
\hline $\mathrm{Q}_{\text {on }}$ & 3143 & 1.0830 & 22.6619 \\
\hline $\mathrm{T}_{\text {off }}$ & 3016 & -9.3621 & 43.1823 \\
\hline $\mathrm{QT}$ & 3016 & -10.3886 & 49.3424 \\
\hline
\end{tabular}

Table 1. Comparison between automatic and manual annotation on all 105 records. Mean and standard deviation measurements are in milliseconds.

Table 1 summarizes the mean and standard deviation of the differences between automatic and manual annotated $\mathrm{Q}$-wave onset $\left(\mathrm{Q}_{\mathrm{on}}\right)$, T-wave offset $\left(\mathrm{T}_{\text {off }}\right)$ and QT interval (QT). Column 2 shows total number of annotated beats that we have compared. The automatic detection algorithm misses some T-wave. From our analysis, the main reason of miss detection is irregular morphology in T-wave. A typical T-wave has bell shape morphology. The algorithm can detect Q-wave onset with reasonable good accuracy and consistency as evident from mean and standard deviation of $\mathrm{Q}_{\mathrm{on}}$. Mean and standard deviation for $\mathrm{T}$-wave offset is larger as expected. T-wave offset is difficult to localize accurately especially for T-wave with very slow repolarization and low signal-to-noise ratio.

\begin{tabular}{|l|r|r|r|}
\hline & Beats & mean & $\begin{array}{r}\text { standard } \\
\text { deviation }\end{array}$ \\
\hline $\mathrm{Q}_{\text {on }}$ & 2372 & 0.8364 & 23.1790 \\
\hline $\mathrm{T}_{\text {off }}$ & 2372 & 3.6071 & 31.3451 \\
\hline $\mathrm{QT}$ & 2372 & 2.7707 & 39.4491 \\
\hline
\end{tabular}

Table 2. Comparison between automatic and manual annotation on 70 selected records. Mean and standard deviation measurements are in milliseconds.

We have developed the device to assess the effect of 
new drug on QT interval. The subjects participating in the new drug trial generally has regular ECG signal. 70 records with high signal-to-noise ratio, regular QRS complex and T-waves morphology are chosen manually from the database for second stage of evaluation. Table 2 summarizes the detection accuracy of the algorithm on selected ECG records. Compare to results in Table 1, the mean and standard deviation in Table 2 are lower. This shows the algorithm detects the Q-wave onset and Twave offset with reasonably good accuracy and stability on regular ECG signals.

\section{Discussion and conclusions}

A smart phone-based QT interval measurement system was developed. The system assists pharmaceutical company in QT prolongation assessment prior to new drug approval. The QT measurement data is transmitted wirelessly to an internet server. Therefore, it is very convenient for the test subjects as they do not need to visit the lab frequently.

The automatic annotation of the system was compared against manual annotation by cardiologist on a QT database. The system localized the Q-wave onset and Twave offset with reasonably good accuracy and stability. However, the system failed to detect Q-wave onset and T-wave offset with irregular ECG morphology. Currently the system utilized only one channel signal. The result can improve further if additional signals are used. In many cases, T-wave offset boundary can be determined clearly from another signal.

The algorithm was implemented on $\mathrm{O}_{2}$ smart phone. The onboard ARM processor can process the ECG data in real-time. We are working on code optimization to reduce computational complexity and reduce the battery power usage.

\section{References}

[1] Kautzener J., QT interval measurements: Card Electrophysiolol Rev 2002;6(3):273-7:63-65.

[2] Jayasinghe R., Drugs and the QTc interval,

[3] Pan J. and Tompkins W. J., A real-time QRS detection algorithm, IEEE Trans. Biomed. Eng., 1985:32(3):220236.

[4] Li C., Zheng C., Tai C., Detection of ECG characteristic points using wavelet transforms. IEEE Trans Biomed Eng $1995 ; 42: 21-8$.

[5] Mallat \& Zhong, Characterization of Signals from Multiscale Edges, IEEE Trans Pattern Analysis and Machine Intelligence, 1992;14(7):710-732.

[6] Cohen A., Kovacevic J., Wavelets: The mathematical background. Proc. IEEE 1996;84:514-522.

[7] Laguna P. R. G. Mark, Goldberger A., Moody G. B., A database for evaluation of algorithms for measurement of QT and other waveform intervals in the ECG, Computers in Cardiology 1997.
[8] Moody G. B., Koch H., Steinhoff U., The PhysioNet/Computers in Cardiology Challenge 2006: QT Interval Measurement, Computers in Cardiology 2006.

[9] Chen X., Xie W., Dong L., Tin Z.K., Sankaranarayanan M, Reliable automated QT interval measurement for clinical evaluation, Computers in Cardiology 2006.

[10] Hayn D., Kollmann A., Schreier G, Automated QT Interval Measurement from Multilead ECG Signals, Computers in Cardiology 2006.

Address for correspondence

Eng Thiam Lim

Institute for Infocomm Research

21 Heng Mui Keng Terrace

Singapore 119613

etlim@i2r.a-star.edu.sg 